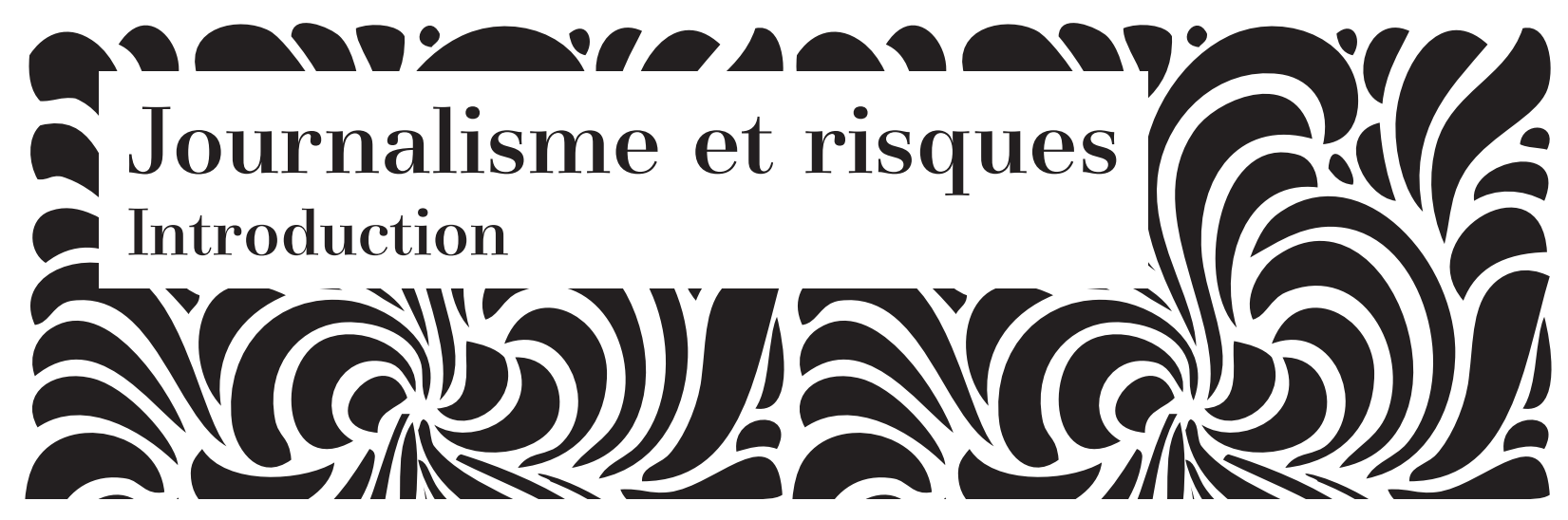

\author{
Francois Demers \\ Professeur \\ Université Laval \\ Canada \\ francois.demers@com.ulaval.ca
}

Renaud de La Brosse

Professeur

Université Linnaeus

Suède

renaud.delabrosse@lnu.se

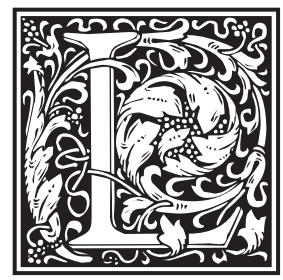

'appel à textes pour ce numéro sur les "dangers et les risques" présentait le journalisme comme un prolongement de la liberté d'informer, en ce que celle-ci suppose non seulement la liberté de chercher l'information de façon systématique et d'en faire une activité professionnelle. Le souci pour la liberté d'expression en public, une préoccupation aussi ancienne que les droits de l'homme, parait d'actualité pour au moins trois raisons :

\title{
Marie-Soleil Frère
}

Chercheure Université Libre de Bruxelles Belgique msfrere@ulb.ac.be

Sylvia Moretzsohn Professora Universidade Federal Fluminense Brasil sylviamoretz@uol.com.br

1. la pratique professionnelle du journalisme a toujours comporté des risques mais elle est aujourd'hui confrontée à d'autres dangers en raison d'un environnement politique et économique instable qui augmente, stimule ou provoque, dans plusieurs points du monde, les environnements socio-politiques marqués par la corruption, les trafics illégaux et immoraux, l'impunité de la violence et l'insécurité physique;

2. la figure du journaliste convoquée n'est pas celle, extrême, du consacré sacerdotal pour lequel il serait normal de vivre en héros et de finir martyr, ou encore celle du citoyen exceptionnel, unique et olympique, mais celle du travailleur spécialisé (salarié ou payé à la pièce) qui accomplit des fonctions considérées en principe comme socialement

Pour citer cet article, to quote this article, para citar este artigo :

François Demers, Renaud de La Brosse, Marie-

Soleil Frère, Sylvia Moretzsohn, « Journalisme et risques. Introduction », Sur le journalisme, About journalism, Sobre jornalismo

[En ligne, online], Vol 7, n¹ - 2018, 15 juin -

June 15 - 15 de junho.

URL : http://www.surlejournalisme.com/rev 
et juridiquement légitimes; or, ce marché du travail ratatine rapidement depuis la fin $\mathrm{du}$ siècle;

3. l'extension de la surveillance généralisée grâce aux technologies du numérique arme des sources publiques et privées de plus nombreuses informations sur le journaliste et ses activités, ce qui les rend capables plus qu'avant d'entraver son travail.

À l'évidence, l'examen sous cet angle de la pratique du journalisme dans le contexte contemporain constitue un chantier très large. De multiples pistes de réflexion peuvent être suggérées et des questions surgissent en abondance. Ainsi, quelles sont les parades que les journalistes peuvent déployer face au banditisme, à la déliquescence et à la corruption des États ? Comment se construisent des alliances avec d'autres acteurs, soucieux de s'opposer à ces évolutions problématiques, afin de permettre l'activité journalistique sans risques inconsidérés ? Quelles formules émergent pour soulager la précarité du journaliste dans une organisation du travail qui transfère sur lui seul l'organisation et les conséquences de ses activités ? Quels usages font les journalistes des moyens électroniques de surveillance mis à leur disposition : drones, géolocalisations, bases de données, etc. ? D’autre part, comment se défendent-ils de l'usage de ces mêmes moyens contre eux ?

Les recherches menées sur ces diverses questions sont encore peu nombreuses, ce qui reflète l'inévitable décalage entre la recherche sur le journalisme et la pratique journalistique quotidienne dans un contexte particulièrement bousculé par des changements en cascades. Toutefois, nous présentons dans ce numéro thématique sept textes qui, chacun à sa façon, montrent que le monde de la recherche a débuté l'observation et la consignation de ces nouveaux risques, tout comme ils rappellent comment, dans d'autres contextes dangereux, le journalisme a pu se monter résilient et développer des tactiques d'évitement et de résistance.

Certains de ces textes contribuent plus particulièrement à l'inventaire des nouveaux dangers. Il en va ainsi de l'étude des attaques armées survenues à Ottawa et à Saint-Jean-sur-Richelieu au Canada en octobre 2014 proposée par Marie-Ėve Carignan, professeure à l’Université de Sherbrooke au Québec. Son travail est emblématique des interrogations éthiques posées aux médias et aux journalistes dans les sociétés occidentales avancées, là où se sont sédimentées sur une longue période des consignes déontologiques détaillées. Plus spécifiquement, Marie-Ève Carignan utilise les acquis jurisprudentiels du Conseil de presse du Québec (CPQ) pour éclairer le comportement déstabilisé des médias lors de ces deux attentats et montrer par là les limites de leur auto-régulation.

De son côté, Chrisanti Giotis, doctorante à la University of Technology de Sydney en Australie, met en lumière les biais que fabriquent pour les étrangers - dont les correspondants des médias - les zones internationalisées, installées dans les pays en crise, de plus en plus retranchées et séparées des communautés locales, et qui sont désignées comme des « aidlands" ou "peacelands ». À ses yeux, ces correspondants sont en quelque sorte " embedded "- au sens où on parle des correspondants de guerre incorporés sur le front dans des régiments de soldats - dans le domaine défini territorialement et intellectuellement par l'action humanitaire et les acteurs dudit développement international. Elle illustre son propos par des entrevues avec six "fixers " et des observations faites dans la ville de Goma en République démocratique du Congo. Les risques de cette imbrication sont considérés par elle comme des difficultés non matérielles et des dangers non-physiques qui remettent en cause la qualité et l'éthique du reportage international, suspecté d'encourager ainsi une connaissance limitée et erronée des pays et des conflits dont ils traitent.

Pour sa part, dans un travail d'histoire qui analyse la couverture médiatique de la guerre portugaise en Angola entre 1959 et 1975, Sílvia Torres, doctorante en Sciences de la communication à la Universidade Nova de Lisboa au Portugal, montre qu'une publication peut parvenir malgré tout, dans certaines limites, à donner de la visibilité à un conflit qui autrement aurait pu passer inaperçu. Par le moyen de la méthode classique de l'analyse du contenu, appliquée aux numéros publiés entre 1961-1974 par le magazine Notícia, une publication hebdomadaire dont le siège était à Luanda, et par des entrevues effectuées avec des professionnels qui travaillaient à cette époque dans les médias d'Angola, Sílvia Torres montre comment les reporters ont utilisé des pratiques professionnelles pour parvenir, malgré la censure, à donner de la saillance à ce conflit.

En un sens, le texte suivant, de Patricia W. Elliott, professeure adjointe à l'École de journalisme de l'Université de Regina en Alberta au Canada, prolonge le précédent en ce qu'il traite du journalisme possible, dans un contexte aussi contraignant qu'une guerre, mais sur une plus longue période encore, dans le cadre d'une dictature militaire féroce. Il s'agit des 50 ans et plus de dictature militaire en ex-Birmanie, connue aujourd'hui sous le nom de Myanmar ou Burma. Son étude décrit les perceptions du risque du point de vue des journalistes eux-mêmes et les raisons pour lesquelles ils ont continué à faire du 
journalisme malgré les menaces d'emprisonnement, d'exil ou de mort. L'auteure décrit en même temps le contexte qui a conduit les journalistes à jouer un rôle important dans l'arrivée d'une phase de démocratisation en 2011. Enfin, sa recherche révèle que sous la surface du contrôle étatique pendant la dictature, on trouve une étonnante diversité d'expressions publiques, dans des médias ethniques, des agences de presse en exil, des journalistes citoyens, des blogueurs et même dans les médias pourtant sanctionnés par l'État militaire.

Suivent trois textes sur l'exercice du journalisme dans un pays d'Amérique du nord formellement démocratique depuis longtemps et davantage depuis 2000 alors que débuta l'alternance des partis politiques à la Présidence : le Mexique. Les deux premiers traitent d'agressions non-physiques et des conditions structurelles insécurisant la pratique du journalisme. Diana Denisse Merchant, doctorante en sciences sociales au Centro de Investigaciones y Estudios Superiores en Antropología Social (Ciesas, Occidente) à Guadalajara étudie la situation en Baja California, l'État le plus au nord du pays sur la côte du Pacifique. Sur la carte, cet État paraît presque détaché du Mexique et faire plutôt partie des EtatsUnis, comme un prolongement dans la mer de la Californie états-unienne. L'auteure prend d'ailleurs acte dès le départ de la diversité des situations régionales du journalisme au Mexique. À propos de la Baja California, elle soutient que les journalistes sont immergés dans des menaces continues d'agression économique, éthique et psychologique, que ces pressions sont constantes et qu'elles reflètent la précarité générale des journalistes au Mexique. Elle s'attache alors à décrire les stratégies développées par les journalistes de la presse écrite pour éviter ces agressions, les contourner et leur échapper. Ces manœuvres, elle les a observées pendant 12 mois en 2014 auprès de différents journalistes de cinq municipalités, observations complétées par 25 entrevues de journalistes et par 5 entretiens avec des chargés de communication des Services de l'État. L'auteure analyse ces données en considérant les journalistes non pas comme des pures victimes qui subissent l'oppression mais plutôt comme des «agents " qui «font avec ».

Víctor Hugo Reyna García, doctorant en sciences sociales au Colegio de Sonora, dans la ville de Hermosillo, s'attarde lui aussi sur les menaces permanentes qui pèsent sur les journalistes mexicains plutôt que sur la «crise sécuritaire " devenue emblématique de ce pays qui a connu des dizaines de meurtres de journalistes au cours des dernières années. Il tourne son regard dans une autre direction, celle de l'insécurité d'emploi, endémique au Mexique. À cette fin, il présente les travaux du so- ciologue allemand Ulrich Beck à propos de la Société $d u$ risque et en particulier son cycle de recherches sur la sociologie du travail, qui pose qu'en ce domaine comme dans les autres, la société contemporaine augmente les risques et l'incertitude. Le doctorant introduit ensuite dans le cadre théorique ainsi défini trois concepts de la sociologie du travail plus classique : la sécurité d'emploi, la qualification et la satisfaction. Enfin, il nourrit cette grille d'analyse à l'aide de références aux travaux empiriques, épars, qui ont examiné les conditions d'exercice du métier de journaliste au Mexique dans les dernières décennies, auxquelles il ajoute des données empiriques qu'il est en train de recueillir dans ses recherches sur le terrain.

Le troisième texte à propos du Mexique, et le dernier de ce numéro, est l'œuvre de trois personnes: Salvador De León Vázquez, chercheur au Département de communication de la Universidad Autónoma de Aguascalientes et deux assistants de recherche au même département, la chercheure adjointe Alejandra Bravo Ponce et l'étudiante à la maîtrise E. Maritza Duarte Alcántara. Leur article présente trois organisations d'entraide mises sur pied par des journalistes qui ont conclu qu'il ne servait généralement à rien de faire appel aux instances gouvernementales, pourtant officiellement proclamées services d'aide, étant donné leur inefficacité avérée. Aussi ont-ils choisi de mettre sur pied leurs propres réseaux qui permettent aux expériences des uns d'inspirer les autres, que ce soit pour prévenir ou éviter les violences physiques, pour faire face aux menaces des sources disposant d'une autorité, aux vexations et représailles des hiérarchies dans les salles de rédaction ou aux dangers du côté de la police, des militaires et des milieux criminels. Ces initiatives d'auto-organisation ont émergé dans trois zones différentes : la ville de México, la ville de Juarez dans l'État de Chihuaha au nord et la ville de Tuxtepec, dans l'Oaxaca au sud de Mexico sur la côte du Pacifique. D'autres organisations d'entraide sont en émergence ajoutent les auteurs. Leur naissance est en lien direct avec le climat d'extrême insécurité qu'a entraîné la guerre aux narcotrafiquants déclenchée par le président Felipe Calderón Hinojosa dès son arrivée au pouvoir en 2006.

L'ordre dans lequel nous présentons ces textes indique la direction vers laquelle les prochains travaux sur les dangers et risques du journalisme seront probablement amenés à s'orienter. En effet, le premier, sur le terrorisme à l'intérieur des pays démocratiques, annonce les contraintes sécuritaires avec lesquelles les journalismes nationaux vont avoir affaire dans les prochaines années. Les trois suivants rappellent, chacun à sa façon, les limites et biais du regard occidental sur le reste du monde que 
ses médias et reporters prétendent déployer en permanence. L'article sur le travail des correspondants internationaux à Goma en République démocratique du Congo évoque à la fois l'enfermement physique croissant de la plupart des correspondants dans les hôtels américains des ghettos internationalisés et sécurisés des zones humanitaires mais aussi leur enfermement moral dans les bonnes intentions des ONG internationales. Ces situations se multiplient au même rythme que les guerres et conflits dans le reste du monde, c'est dire que le journalisme y connaît les restrictions de la censure pour cause de conflit armé, en plus de celles qui se déploient dans les dictatures ou les régimes pudiquement qualifiés d'autoritaires. Quant aux trois articles portant sur le Mexique, ils paraissent aborder une conception plus traditionnelle du risque encouru par les journalistes, mais, dans le contexte actuel, ils font en quelque sorte écho au premier texte sur le terrorisme. Le Mexique vit depuis fort longtemps dans un régime de sécurisation armée. Dès lors, la façon dont les journalistes y survivent et $\mathrm{y}$ fonctionnent préfigure peut-être comment les professionnels d'ailleurs et de demain, y inclus dans les pays démocratiques occidentaux, seront forcés d'apprendre à naviguer, à ruser, à composer face au danger. L'expérience mexicaine pourrait être exemplaire et utile dans un contexte où le journalisme semble fondamentalement déstabilisé, même dans les régimes démocratiques. 